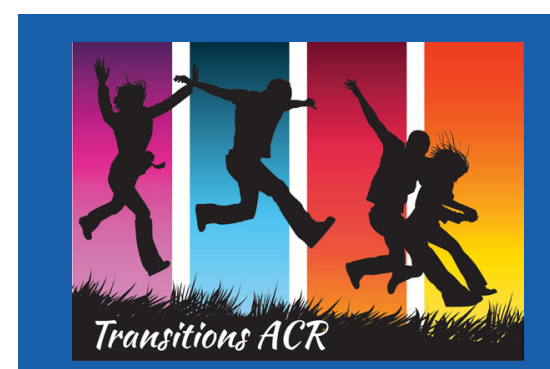

Tip Sheet 14

\title{
Tips and Tricks to Starting a Young Adult Council Part 1: 10 Steps to Starting a Young Adult Advisory Council
}

Transitions ACR

September 2018

\section{What is a Young Adult Advisory Council for Mental Health?}

Y

oung adult advisory councils are groups of likeminded young adults who come together on a consistent basis to share resources and advise organizations on how to better serve and support young people. By incorporating young adult expertise into the design and delivery of mental health services, these councils provide direct feedback on how to improve services and make them more relevant to young adult culture. These steps are based on the Transitions to Adulthood Center for Research's own experience with creating a Youth Advisory Board (YAB). Learn more about our work in the Resources below.

\section{Councils can:}

Educate providers on how to better engage and retain young adults in services

Ensure services are young adult friendly ${ }^{1}$

Give young adults an ongoing opportunity to flex their leadership and advocacy skills

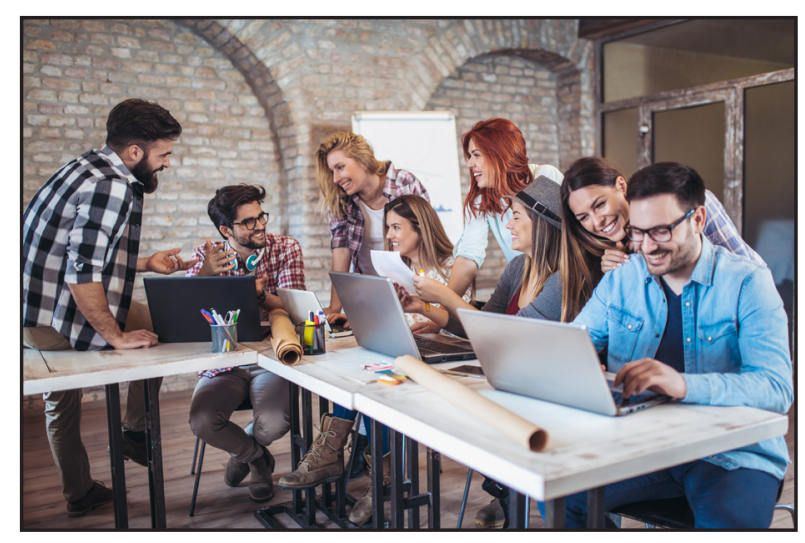

\section{How Do You Start One?}

This tip sheet will walk you through ten steps of developing a young adult mental health advisory council.

Step 1:

Get Top-Down and Bottom-Up Buy-In

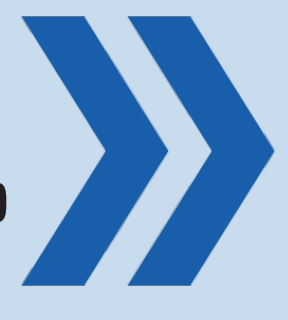

Leaders and staff within the organization must believe in the mission of the council and hold staff accountable to help create youth-infused services. Providing trainings to staff at all levels of an organization can help everyone better understand what the purpose of the council is, as well as the benefits a council can provide to the organization and individual providers.
Step 2:

Establish Mission, Vision, \& Initial Goals of the Council

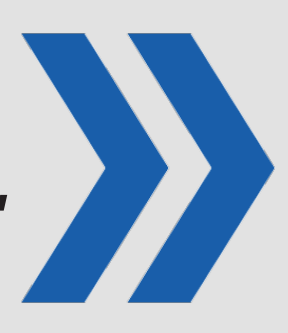

In collaboration with young adult members, organizations should have some primary and secondary goals outlined to set the structure and purpose of the council in the beginning stages. In time, develop the council's mission and vision as well.

\section{Example Primary Goals:}

Improve how mental health services are delivered at the sponsoring organization(s) to make them more appropriately geared toward young adults Increase access to outpatient services for young adults age 16-25

\section{Example Secondary Goals:}

Develop young adult friendly marketing materials Improve mental health awareness in the local community 


\section{Step 3: \\ Plan Council Meeting Times}

It is important that council meeting times accommodate the busy schedules of young adult members; oftentimes, young adults are balancing school and work simultaneously along with other priorities. Be flexible and consider having council meetings after work hours or on weekends when possible.

\section{Quick tip!}

Use free online scheduling platforms like Doodle Polls (www.doodle.com) to find a time that works best for your members.

\section{Step 4: \\ Set a Positive Meeting Location}

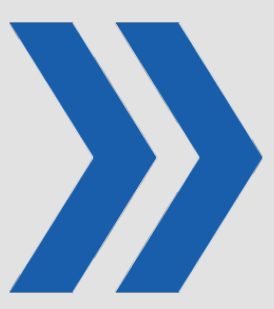

Where you choose to host your meeting can make or break attendance.

Have your meeting in a welcoming space:

$\rightarrow$ Avoid mental health service buildings (e.g. a hospital or outpatient clinic) - these locations can be uninviting and even distressing for members who have had negative experiences seeking mental health treatment.

$\rightarrow$ Book a room at a location in the community (a space without a lot of foot traffic like a local public library, a community center or a bookstore often work out nicely).

Many young adults struggle with access to reliable transportation, so consider the following:

$\rightarrow$ Choose a location on a public transit route

$\rightarrow$ Provide transportation stipends or bus passes

$\rightarrow$ Host virtual meetings with video conferencing software

Step 5:

Recruit Members
Developing membership can be challenging, try these tips:

- Have a written description of a council member's role to share when recruiting members, this helps set clear expectations. Include the following in the description:

$\rightarrow$ Age requirements (e.g. 18-30 years; $16-25$ etc.)

$\rightarrow$ Expectation of disclosure: members should be explicitly welcomed to share details about their experience living with a mental health condition, but should not be required to do so

$\rightarrow$ Goals of the council \& draft form of mission/vision

$\rightarrow$ Time commitment

$\rightarrow$ Training/guidance provided

$\rightarrow$ Compensation offered

- Consider multiple advertising avenues (e.g. mental health listserv, social media) to recruit young adults from many walks of life with various recovery experiences. Diversity is a key piece of a council since lived expertise can vary greatly from person to person.

- Sending an advertisement (e.g. flyer) and a letter of request for recommendations to colleagues within and outside of your organization to help identify young adults who are a good fit for your council.

- Recruitment is likely to be continuous since young adults' lives change so frequently, it is common for councils to have members joining and moving on from the council frequently. 


\section{Step 6: Train Members and Invited Speakers}

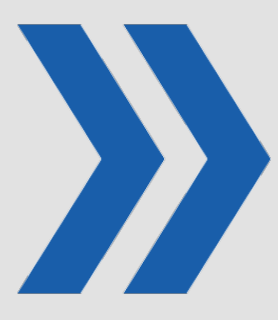

Member training: Hold a training call or meeting for new members to learn about the council and the history of the organization that houses the council. Other training topics can be: how to contribute in respectful discussions or strategically share your recovery story.

Speaker training: Educate any adult guests/speakers on how to be 'silent supporters' at meetings and how to encourage council members to provide feedback.
Step 7:

Develop a Meeting Structure

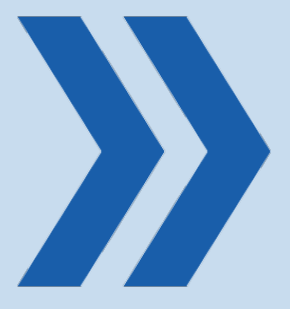

Setting a comfort clause: A comfort clause is a list of rules for council members and guests to follow to ensure the meeting is a safe space. We highly encourage the leader(s) of the council to create a comfort clause in collaboration with council members. Moving forward, this comfort clause should be consistently enforced by both council leaders and members.

Some examples of items that young adults have added to comfort clauses:

$\rightarrow$ Using person-first language (person living with schizophrenia as opposed to "a schizophrenic")

$\rightarrow \quad$ Using agreed upon terms (like mental health condition instead of illness)

$\rightarrow$ Supporting open honesty even when opinions differ

$\rightarrow$ Vegas confidentiality rule: What is said during a meeting stays in a meeting and only with member approval can what is said in a meeting be shared outside the council

Develop the agenda: Provide an opportunity for members to identify what topics they would like to see covered. The agenda should also always provide a reminder of the group mission and comfort clause. It should include time for socialization (i.e. icebreakers) and a break.

\begin{tabular}{|l|l|}
\hline \multicolumn{2}{|l|}{ Sample Agenda } \\
\hline 2:00 - 2:15 & $\begin{array}{l}\text { Introductions and Opening Icebreaker (e.g. “If you could invite three well known } \\
\text { people from history to a dinner party, who would you choose and why?") }\end{array}$ \\
\hline 2:15 - 2:30 & Updates and announcements from group members/group leaders \\
\hline 2:30 - 3:00 & Providing feedback on organization brochure \\
\hline 3:00 - 3:10 & Snack Break Social! \\
\hline 3:10 - 3:40 & Discussion: How do we better advertise our services to young adults? \\
\hline 3:40 - 3:55 & Closing Icebreaker: Highlights and appreciations of the week \\
\hline
\end{tabular}

Step 8:

Running the Meeting

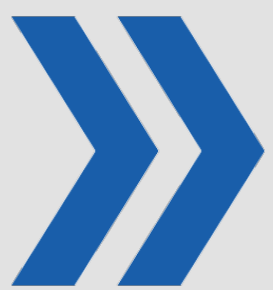

Identify a facilitator to lead each meeting, help moderate discussions and walk the group through the agenda. If possible, the facilitator(s) should be young adults themselves and employed by the organization hosting the council. Facilitation requires availability and effort outside scheduled council meetings, so its helpful if the facilitator is employed by the organization as they may have structured time available to plan for the meeting.

Include feedback on the mission/vision from council members. They should be encouraged to take ownership of the council and should be involved in finalizing the mission and vision to ensure their voices are represented in the core structure of the group

MAKE IT FUN: Keeping the council fun and enjoyable can be a great way to make young adults comfortable. Offer time (e.g. scheduled breaks) for socializing. Starting and ending meetings with icebreakers and/or highlights and appreciations of the week at the beginning to provide a fun space for non-business-related conversation. 


\section{Step 9: Develop Engagement Strategies}

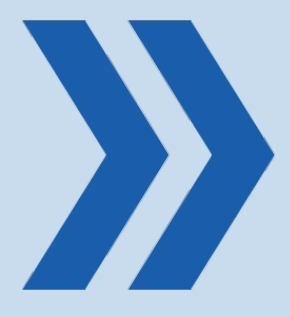

- Provide guidance and positive feedback: This lets young adult members know how to be most helpful and effective in their role. This can be done formally during regular one-on-one check-ins or informally during the council meetings.

- Provide Consistent Reminders:

Young adults are busy and appreciate receiving email or text reminders about upcoming council meetings.

- Membership Renewal: Routinely check in with council members (e.g., every 6 months) to see if members would like to renew their membership with the council. This helps provide a loose but concrete commitment, while also providing a space for members to revisit their involvement and consider moving on to new opportunities without guilt.

\section{Step 10: Talk the Talk}

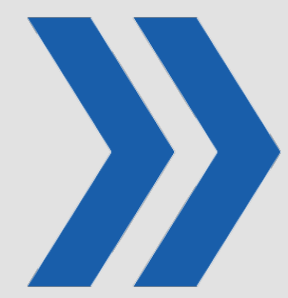

After receiving young adult feedback on a subject, showcase how their feedback was incorporated. Showing a finished product or how their feedback helped shape services will keep young adults engaged and motivated to continue their membership activities. This shows that their feedback is valued and their involvement is meaningful.

\section{Resources}

Mizrahi, R. \& Duperoy, T. (2018). Tips and tricks to starting a young adult council [Video webinar and PowerPoint slides].

Retrieved from https://www.umassmed.edu/TransitionsACR/publication/webinars

Costa, A. \& Duperoy, T. (2016). Nothing about us without us: growing meaningful young adult involvement in your organization [Video webinar and PowerPoint slides]. Retrieved from https://www.umassmed.edu/TransitionsACR/publication/webinars

\section{References}

1. Kirby, P., Lanyon, C., Cronin, K., \& Sinclair, R. (2003). Handbook - Building a culture of participation: Involving children and young people in policy, service planning, delivery and evaluation. Nottingham: Department for Education and Skills.

\section{Suggested Citation}

Mizrahi, R., Costa, A., \& Youth Advisory Board of the Transitions ACR. (2018). Tips and Tricks to Starting a Young Adult Council Part 1: 10 Steps to Starting a Young Adult Advisory Council. Worcester, MA: University of Massachusetts Medical School, Department of Psychiatry, Systems and Psychosocial Advances Research Center (SPARC), Transitions to Adulthood Center for Research.

\section{Visit Transitions ACR online at https://www.umassmed.edu/transitionsACR}

The contents of this tip sheet were developed under a grant with funding from the National Institute on Disability, Independent Living, and Rehabilitation Research, and from the Center for Mental Health Services of the Substance Abuse and Mental Health Services Administration, United States Department of Health and Human Services (ACL Grant\# 90RT5031, The Learning and Working Transitions RRTC). NIDILRR is a Center within the Administration for Community Living (ACL), Department of Health and Human Services (HHS). The contents of this tip sheet do not necessarily represent the policy of NIDILRR, ACL, HHS, or SAMHSA and you should not assume endorsement by the Federal sheet do not 